\title{
Research on the Method for Periodic Estimation of the PN Sequence in the Lower SNR DS/SS Signals
}

\author{
Fan Zhou, Yongxin Feng* and Xiaoyu Zhang \\ Shenyang Ligong University, Shenyang, 110159, China \\ *fengyongxin@263.net
}

\begin{abstract}
The periodic estimation method of the PN sequence in DS Signal is researched. It is hard for traditional methods to estimate PN period of DS/SS accurately in the condition of low Signal-to-noise ratio. On the basis of the traditional spectrum reprocessing and the cepstrum method, a PN period estimation method which has integrated serial average with spectrum reprocessing and the cepstrum are put forward to solve this problem and meet the requirements of lower Signal-to-noise ratio. The simulation results show that, when the period of PN sequence is estimated accurately, the cepstrum method has better estimating performance than the spectrum reprocessing method, and the spectral estimation method has lower Signal-to-noise ratio tolerance after improvements.
\end{abstract}

Keywords: DS/SS, Periodic estimation of the PN sequence, Serial average method, Spectrum reprocessing, Cepstrum

\section{Introduction}

DS signal buried in noise propagation, making it difficult to distinguish with noise, has obvious immunity and anti-interception nature [1-2]. Since it produces, people have been looking for the detection and parameter estimation methods. Pseudo code estimate of signal is one of the parameters of signal estimate, but also a necessary prerequisite for the completion of other parameters, providing the necessary data for post-processing. The higher-order spectral method in the literature [3] mentioned a larger amount of data to achieve an accurate estimate of the pseudo-code period, and it is susceptible to noise, remains to be improved to work at lower SNR. Correlation accumulation method in the literature [4] used delaying auto-correlation to detect, convergence rate is slow in low SNR and is also susceptible under noise. Pseudo-code cycle estimation algorithm based on second-order distance in the literature [5] mentioned, which determines the accuracy of the estimated initial phase error estimate greatly affected by the noise.

These pseudo-code cycle estimation method under low SNR conditions are difficult to estimate pseudo-code cycle exactly, This paper studies the problem for this estimate, Put forward a set of pseudo-code cycle spectrum estimation method which combines two times spectroscopy, average method and cepstrum wears. The method improved based on the traditional spectrum estimation method and its advantage is that it can successfully reduce the Signal-to-noise ratio tolerance of pseudo-code cycle estimation.

Firstly, this paper described the traditional method of secondary spectrum and cepstrum, then the key to make a deep analysis on set average method theory of improved method, and finally simulated, estimate and analysis for the improved method.

${ }^{*}$ Corresponding Author 


\section{Theory About Spectral Estimation of DSSS Signal Pseudo Code Period}

\subsection{Traditional Secondary Spectrum Theory}

The auto-correlation function (ACF) of the DSSS signals $s(t)$ can be expressed as:

$$
R_{S}(\tau)=\frac{1}{T} \int_{-T / 2}^{T / 2} s(t) s(t-\tau) d t=\frac{1}{2} R_{d}(\tau) \cos \omega_{0} \tau
$$

Where, $a(t)$ is the information code, $c(t)$ is the PN code, and they are independent with each other, so, $R_{d}(\tau)=R_{a}(\tau) R_{c}(\tau)$, where $R_{a}(\tau)$ and $R_{c}(\tau)$ repents the ACF of $a(t)$ and $c(t)$, which can be expressed as:

$R_{c}(\tau)=\left\{\begin{array}{cc}1 & \tau=0 \\ -1 / N & \tau \neq 0\end{array}\right.$

The power spectrum density (PSD) can be expressed as following after FFT of the $R_{c}(\tau):$

$G_{c}(\omega)=\frac{1}{N^{2}} \delta(\omega)+\frac{N+1}{N^{2}} S a^{2}\left(\frac{\omega T_{c}}{2}\right) \sum_{\substack{k=-\infty \\ k \neq 0}}^{\infty} \delta\left(\omega-\frac{2 k \pi}{N T_{c}}\right)$

From Formula (3) can be seen that the PSD of the PN code is scatter lines with the period of $\omega_{1}=2 \pi /\left(N T_{c}\right)$. Furthermore, according to the convolution theorem, the PSD of the DSSS signals can be expressed as:

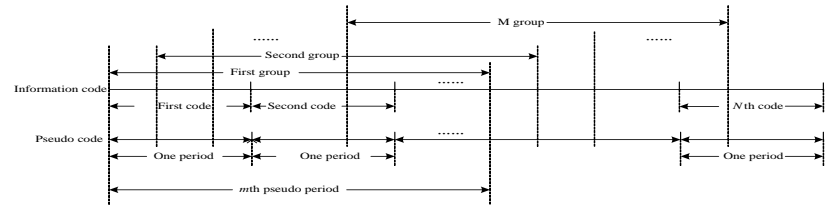

Take formula (3) into (4), and when the PSD is single, the PSD of DSSS signals can be expressed as:

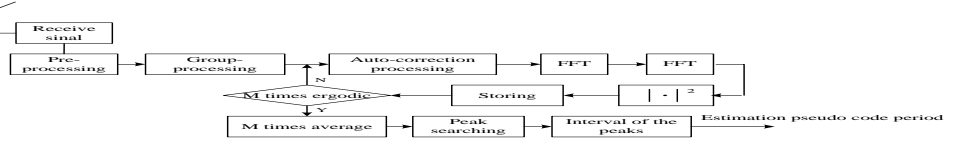

Formula (5) can be simplified as:

$G_{s}(f) \cong \frac{1}{2 N} \sum_{l=-\infty, l \neq 0}^{\infty} \sin c^{2}\left(\frac{\pi l}{N}\right) \delta\left(f-f_{0}-\frac{l}{N T_{c}}\right)$

From Formula (6) can be seen that the scatter lines period of the DSSS signals in PSD is $1 / N T_{C}$, and $\mathrm{N} \gg>1$.

So-called traditional secondary spectrum method [6-10] is that look the power spectrum of the signal as the input signal to solve power spectrum again. Secondary method of traditional theory of power spectrum signal autocorrelation function is 
calculated on the basis of autocorrelation function, then finds the Fourier transform of the autocorrelation function and gets the power spectrum of a signal.

Concluded that signal traditional secondary method of expression can be expressed as:

$$
\begin{aligned}
& G_{s}(e)=\left|F T\left[G_{s}(f)\right]\right|^{2} \\
= & \left|F T\left[\frac{1}{2 N} \sum_{k=-\infty, k \neq 0}^{\infty} \sin c^{2}\left(\frac{\pi k}{N}\right) \delta\left(f-f_{0}-\frac{k}{N T_{c}}\right)\right]\right| \\
= & \left|\frac{T_{c}}{2} \sum_{k=-\infty}^{\infty}\left(1-\frac{\left|e-k N T_{c}\right|}{T_{c}}\right)\right|^{2},\left|e-k T_{c}\right| \leq T_{c}, k=0, \pm 1, \pm 2, \cdots
\end{aligned}
$$

Which $f_{0}$ can be expressed as carrier frequency, $N$ can be expressed as pseudo code cycle, $T_{c}$ can be expressed as Chepstow width, $G_{s}(f)$ can be expressed as power spectrum of Direct sequence spread spectrum signals. From (7) expression we know that direct sequence spread spectrum signal after processing of power spectrum for secondary spectrum, signal energy is gathered in some of the more pointed triangle pulse, pulse spacing is an integer multiple of pseudo-code cycle, Gaussian white noise power spectrum after passing through the secondary spectrum processing do not have this feature. Therefore, the spacing between adjacent triangles by measuring pulse sequence can be estimated PN cycle [11-19]. Due to the power spectrum has done secondary processing, the $e$ of formula (1) actually having a dimension of time.

\subsection{Traditional Cepstrum Theory}

The so-called traditional cepstrum is the logarithm of signals' power spectrum which using an input signal to solve power spectrum again. Traditional cepstrum of signal expression can be expressed as:

$$
\begin{aligned}
& c(\tau)=\left|F F T\left[\log |F F T[s(t)]|^{2}\right]\right|^{2} \\
& \cong T_{1}^{2}(\tau)+\left[T_{1}(\tau) \sum_{k=-\infty}^{\infty} \delta\left(\tau-k N T_{c}\right)\right]^{2}
\end{aligned}
$$

The formula (8) shows that the signal after passing through the cepstrum transform and received direct sequence spread spectrum signal of pulse outputs, the interval between pulses to PN integer multiple of the cycle length. Therefore, signal pseudo code period can be estimated by the spacing between adjacent pulses.

\subsection{Improved Spectral Estimation Theory}

Improved spectral estimation methods include the traditional quadratic GC improvements as well as improvements to traditional cepstrum. Set-average method is the key to improved spectral estimation, the essence is to group the direct sequence spread spectrum signals received, and then the secondary spectrum and cepstrum is estimated for each set of signals completion, final estimates to group all estimates of the arithmetic mean value of the results as the final result. The method of set-average working principle is shown in Figure 1.

As Figure 1, shows, the average law requires each group of signal set containing $\mathrm{m}(\mathrm{m}>=2)$ pseudo code period, and the signal length of $\mathrm{m}$ groups is as same. Combination of the quadratic spectrum and cepstrum theory can be easy to know that representative of each set data containing the information about the signal which is existing in the sharp pulse, and each mixed noise set data can be considered to be statistically independent for each other. When these set data through m-times average, the noise variance will be 
reduced from the original to $\delta^{2} / M$, but representatives of sharp pulse amplitudes after average remained unchanging, so the algorithm improves the signal to noise ratio, thereby improving the estimation accuracy of pseudo code period.

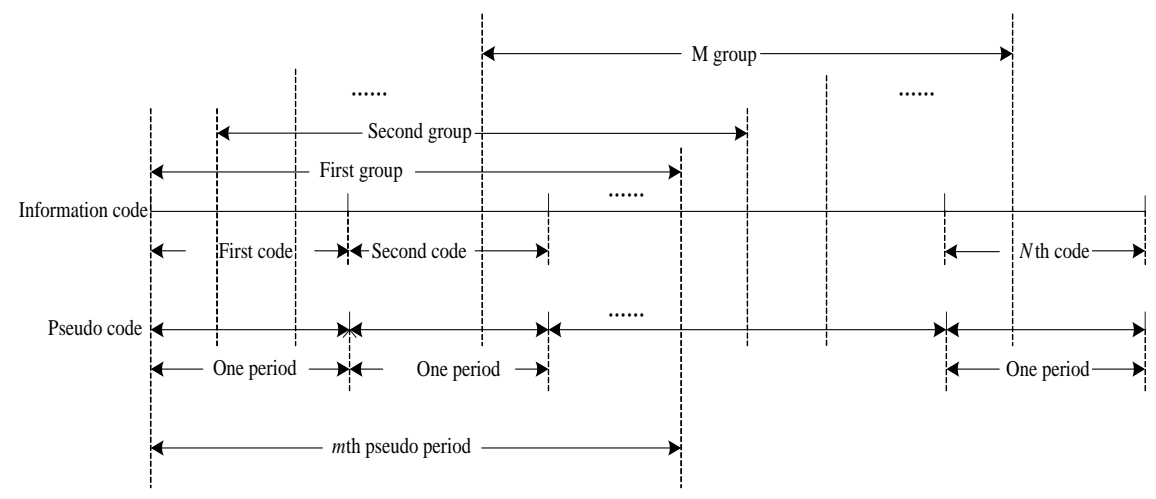

Figure 1. Working Principle of Ensemble Average Method

Spectrum estimation based on the traditional theory, which is shown in Figure 2, and Figure 3, inflect the principles of two improved spectral estimation method. The improved cepstrum method is increased the log-processing compared with the improved secondary spectrum method, both processing are similarly. Taking the improved secondary spectrum method as an example, the working processing is as following.

Step1: The pre-processing signal is divided into more than one group, then calculate the auto-correlation function of each signal;

Step2: Using the Fourier transform of the auto-correlation function of each signal can get the signal's power spectrum;

Step3: Power spectrum of each group of signal on the Fourier transform again, then get the square of the modulus, furthermore, get the quadratic signal power spectrum;

Step4: Through quadratic spectrum of all group signals to linear average and peak searching, the PN code period can be estimated according to peak interval.

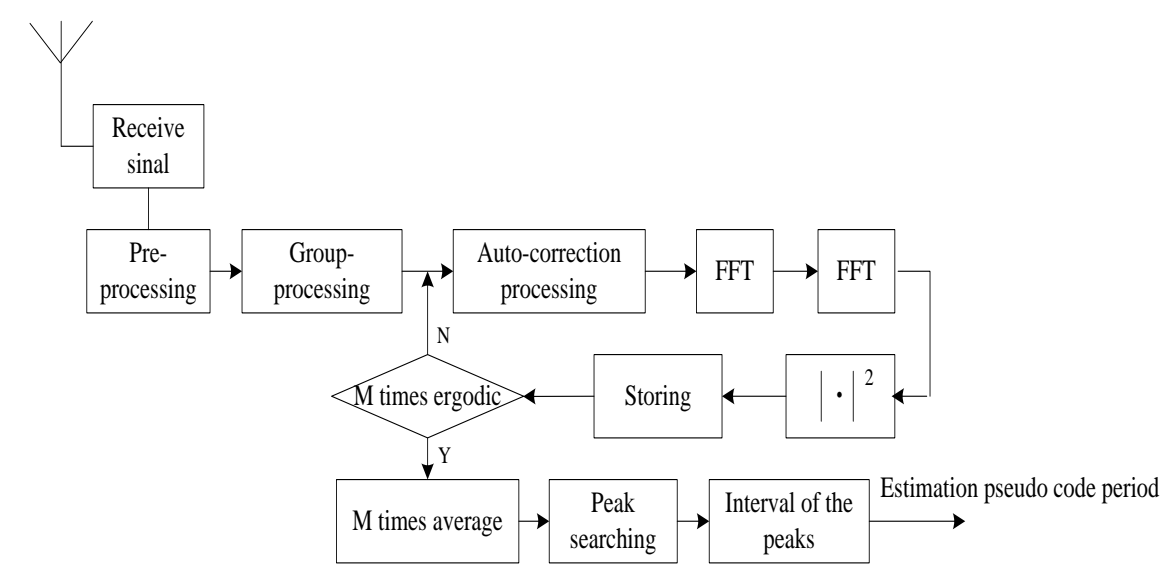

Figure 2. Working Principle of Improved Quadratic Spectrum Method 


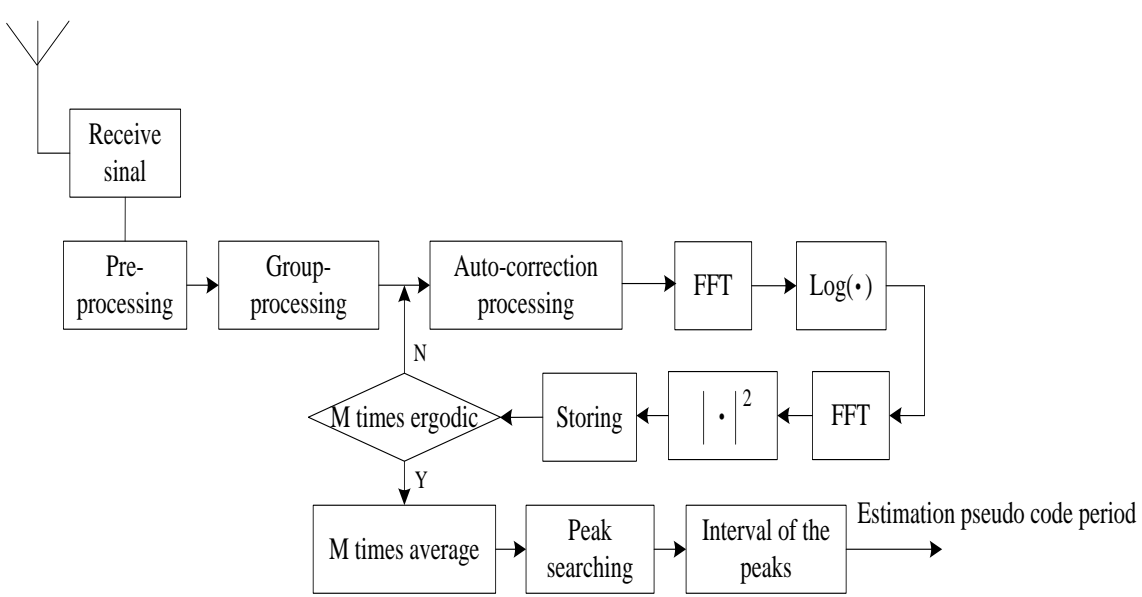

Figure 3. Working Principle of Improved Cepstrum Method

\section{Simulation and Estimation Performance Analysis}

\subsection{Quadratic Spectrum Estimation Results}

In order to verify the feasibility and correctness of both traditional and improved quadratic spectrum method, using MATLAB for simulation tools to complete the estimation of DSSS signal PN code period. During the testing of two methods, same simulation parameters is select: The carrier frequency is $20 \mathrm{MHz}$, information rate is $10 \mathrm{KHz}$, pseudo code rate is $R_{b}=2.55 \mathrm{MHz}$, pseudo-code is $T=0.1 \mathrm{~ms}$, sampling rate is $S a=6$ bit/chip, number of group $M=350$. Each group included 10 cycles pseudo code, and one period of pseudo code only modulate one bit information code.

Figure 4 shows the pseudo code period estimation results of the traditional quadratic spectrum method which SNR is equal to the $-9 \mathrm{~dB}$. From the figure can be shown that the traditional quadratic algorithm can get a group of distinct peak values, further calculation showing that the peak corresponds to the sampling points is 22952, two defective peaks corresponding to the sample point is 24482 and 26012, thus the peak spacing $D$ is $24482-22952=26012-24482=1530$.

Taking the real Pseudo-code cycle length into account, and one chip concluding six sampling points, so true Pseudo-code corresponding points is $3.6 L=S a \times N=1530$, as Figure 4, shows that Pseudo-code cycle is 1530 sampling points, which is equal to $1530 / 6=255$ chips, can indicate the method of estimation is successful .

Figure 5, shows the pseudo code period estimation results of the improved method of quadratic spectrum method which SNR is equal to the $-10 \mathrm{~dB}$. It can be known form Figure 5, that peak spacing $D$ is $15302-13772=16832-15302=1530$, which is also equal to the actual pseudo-code cycle $N$, so the estimation method of PN code period is also successful. 


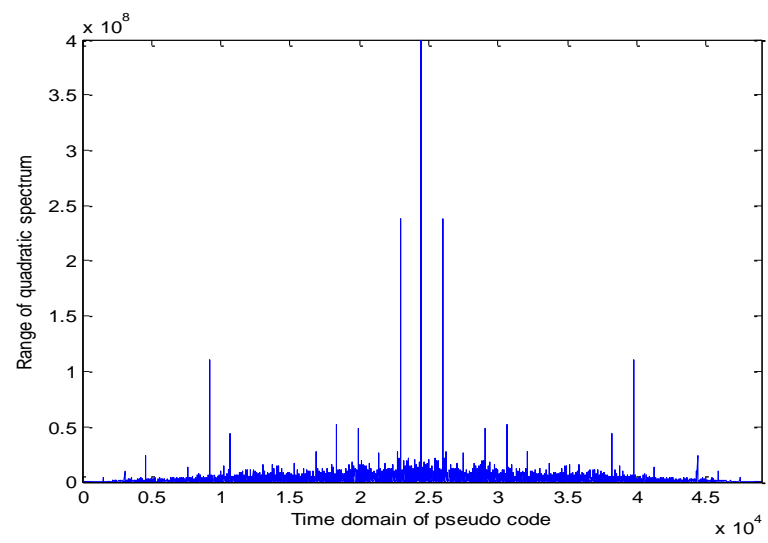

\section{Figure 4. Estimation Effect Drawing of Traditional Quadratic Spectrum} Method

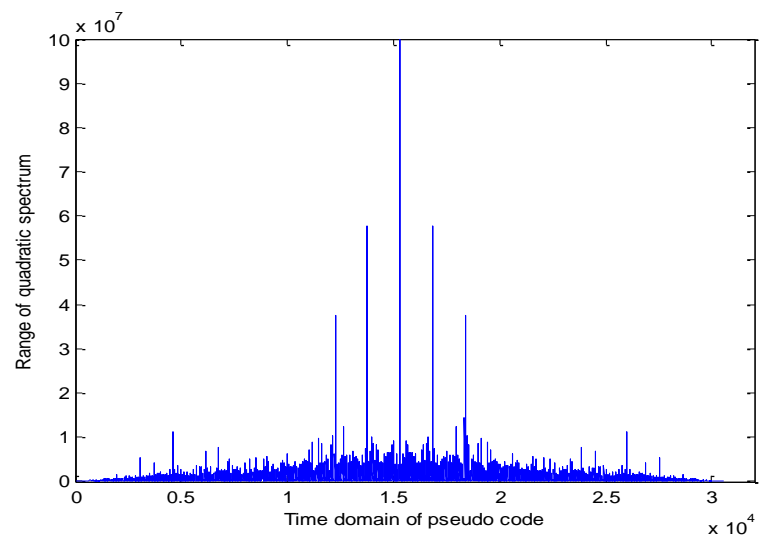

Figure 5. Estimation Effect Drawing of Quadratic Spectrum Method

\subsection{Cepstrum Estimation Results}

Select the same parameters in the quadratic spectrum to test the estimation results in both traditional cepstrum method and improved cepstrum methods. Figure 6, shows the pseudo code period estimation results of the traditional cepstrum method which SNR is equal to the $-10 \mathrm{~dB}$. It can be seen clearly form Figure 6 , that the cepstrum peak line is 9182, 10712 and 12242. Therefore, peak spacing is $D=10712-9182=12242-10712=1530$.

The actual pseudo code period is $N=R_{b} \times T=255$ chips, and the true pseudo code period sampling points is $L=S a \times N=1530$, as Figure 6 shows that Pseudo-code cycle is 1530 sampling points, which is equal to $1530 / 6=255$ chips, can indicate the method of estimation is successful .

Figure 7, shows the pseudo code period estimation results of the improved cepstrum method which SNR is equal to the $-11 \mathrm{~dB}$.It can be known form Figure 7, that peak spacing $D$ is $D=7652-6122=9182-7652=1530$, which is also equal to the actual pseudo-code cycle $N$, so the estimation method of PN code period is also successful. 


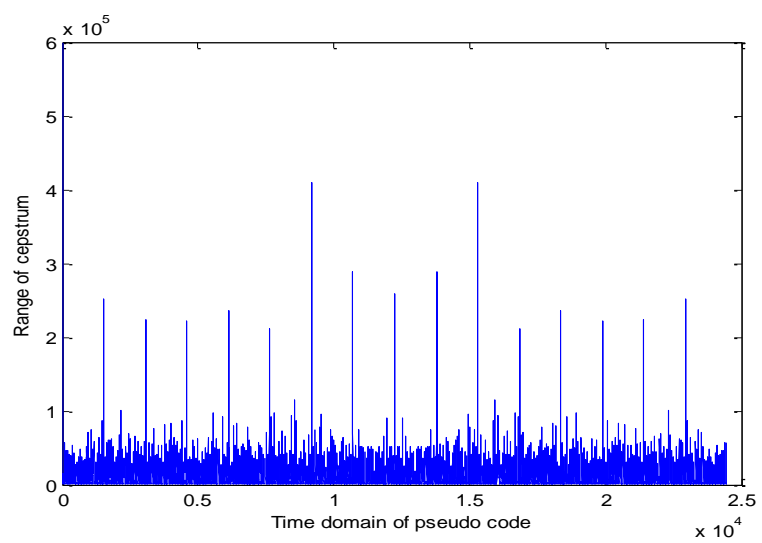

Figure 6. Estimation Effect Drawing of Traditional Cepstrum Method

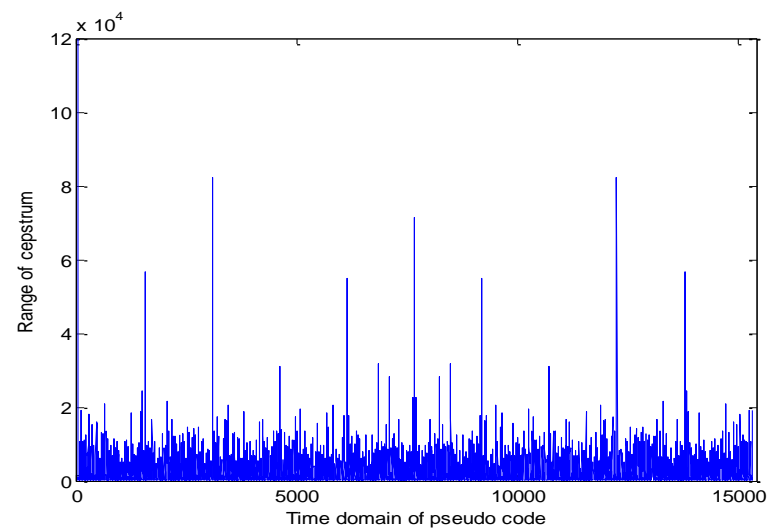

Figure 7. Estimation Effect Drawing of Improved Cepstrum Method

\subsection{Estimation Performance Analysis}

In order to see the estimation performance of the method, using the same parameters exclude the PN code length which is $31,255,511,1023$ to analysis the performance of DS/SS signal estimation in-depth.

Signal-to-noise ratio tolerance of pseudo-code cycle varies with the code length using he traditional and improved quadratic spectrum method can be successfully estimated in Figure 8. It can be shown that the improved quadratic spectrum method can achieve the lower Signal-to-noise ratio tolerance compared with the quadratic spectrum method. With the increasing length of the pseudo code, SNR tolerance is becoming lower and lower.

Signal-to-noise ratio tolerance of pseudo-code cycle varies with the code length using he traditional and improved cepstrum method can be successfully estimated in Figure 9. It can be shown that the improved cepstrum method can achieve the lower Signal-to-noise ratio tolerance compared with the cepstrum method. With the increasing length of the pseudo code, SNR tolerance is becoming lower and lower.

Signal-to-noise ratio tolerance of pseudo-code cycle varies with the code length using the traditional quadratic spectrum and cepstrum method can be successfully estimated in Figure 10. It can be shown that each method achieves the pseudo-code estimation of DSSS signal. Compared with the method of traditional cepstrum, the traditional quadratic spectrum method has a lower SNR tolerance. With the increasing length of the pseudo code, all of the SNR tolerances are gradually decreasing.

Signal-to-noise ratio tolerance of pseudo-code cycle varies with the code length using the improved quadratic spectrum and cepstrum method can be successfully estimated in Figure 11. It can be shown that the final estimation results are not affecting when 
combined with the ensemble-average method. Compared with the method of improved quadratic spectrum, the improved cepstrum method has a lower SNR tolerance. With the increasing length of the pseudo code, all of the SNR tolerances are gradually decreasing.

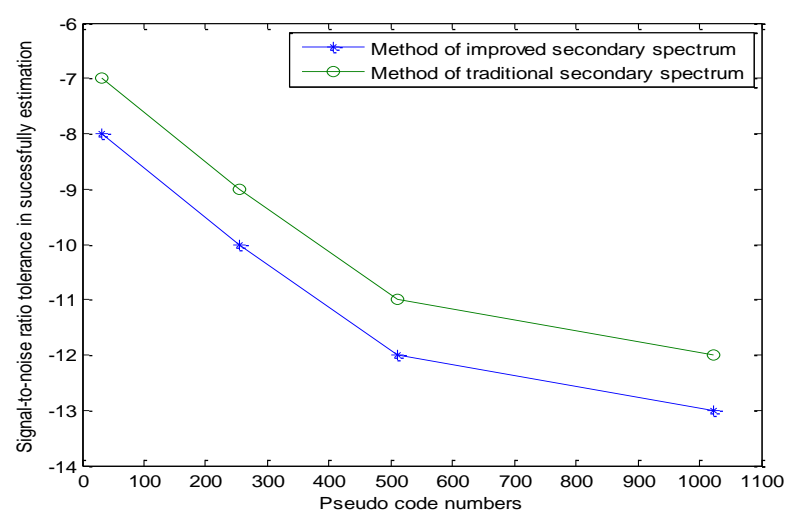

Figure 8. Estimation-Performance Curve of Quadratic Spectrum Method

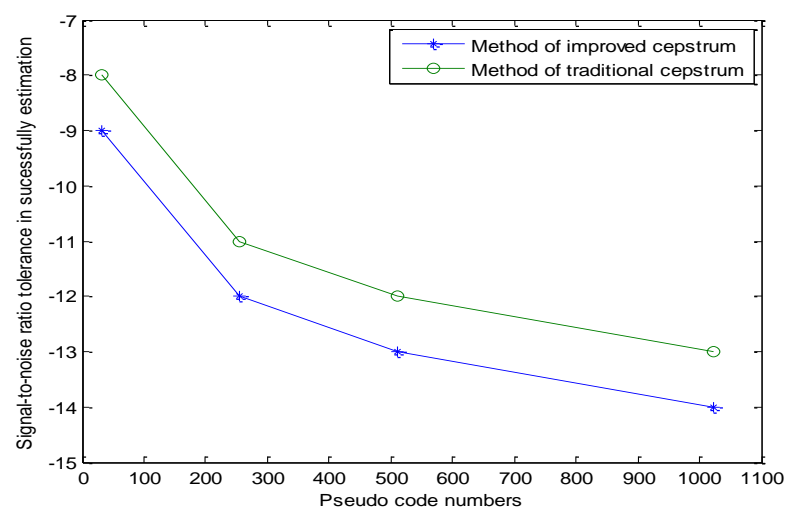

Figure 9. Estimation-Performance Curve of Cepstrum Method

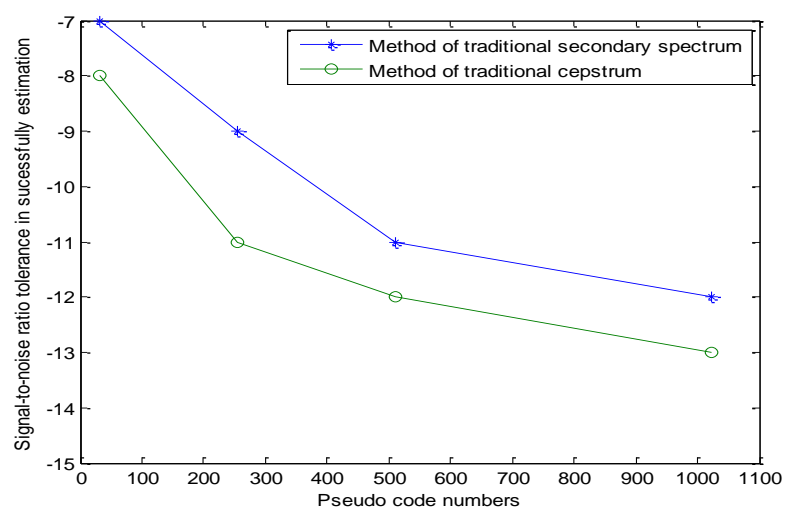

Figure 10. Estimation-Performance Curve of Traditional Quadratic Spectrum Method and Traditional Cepstrum Method 


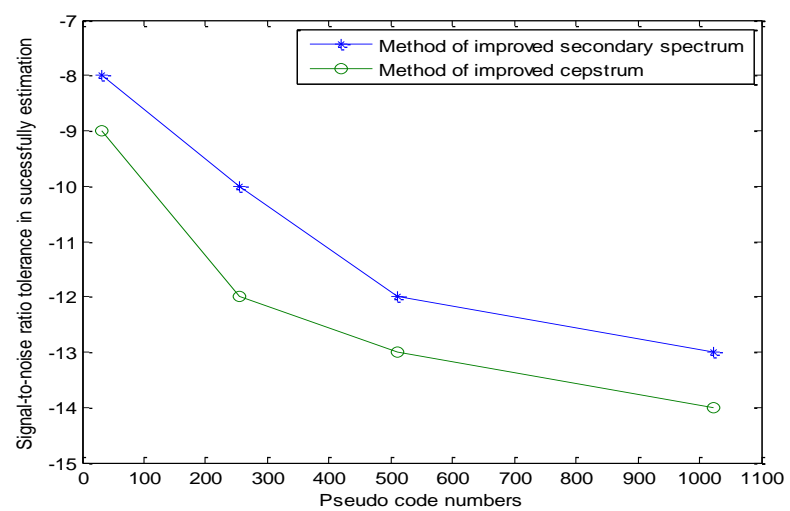

\section{Figure 11. Estimation-Performance Curve of Improved Quadratic Spectrum Method and Improved Cepstrum Method}

\section{Conclusion}

Theoretical analysis and simulation results show that traditional method can realize the DSSS pseudo code period. The SNR tolerances of the improved algorithms is lower than traditional method in the same conditions, in other words, the method of quadratic spectrum and cepstrum method has better performance. With the increasing length of the pseudo code, SNR tolerance is becoming lower and lower. Cepstrum method is better than the quadratic spectrum, which does not require correlation function. Cepstrum method is a algorithm which computational is simple, and can be used in real-time situations.

\section{Acknowledgments}

It is a project supported by New Century Program for Excellent Talents of Ministry of Education of China (NCET-11-1013), the National Natural Science Foundation of China (No. 61501309), the China Postdoctoral Science Foundation (No. 2015M580231), and the Program of Liaoning Excellent Talents in University (No. LJQ2014022).

\section{References}

[1] D. Adamy, "Jamming Direct Sequence Spread Spectrum Signals", Journal of Electronic Defense, vol. 32 , no. $10,(\mathbf{2 0 0 9})$, pp. 64-66.

[2] M. K. Tsatsanis and G. B. Giannakis, "Blind estimation of direct sequence spread spectrum signals in multipath", IEEE Transactions on Signal Processing, vol. 45, no. 5, (1997), pp. 1241-1252.

[3] Z. J. Zhao and J. J. Wu, "The study on the detection methods of DSSS/QPSK signal based on the fourthorder cumulant", ACTA Electronica Sinica, vol. 35, no. 6, (2007), pp. 1046-1049.

[4] T. Q. Zhang, Z. Z. Zhou and Y. M. Kuang, "New method for periodic estimation of PN sequence in the lower SNR long code DSSS signals", Systems Engineering and Electronics, vol. 29, no. 1, (2007), pp. 12-16.

[5] H. Sato and T. Ohtsuki, "Frequency domain channel estimation and equalization for direct sequence ultra wideband (DS-UWB) system”, Communications, IEEE Proceedings, vol. 153, no. 1, (2006), pp. 93-98.

[6] J. Zhu, B. Chen and B. Tang, "Fast detection and parameter estimation method for multi-component LFM signal”, Journal of Electronic Measurement and Instrument, vol. 22, no. 1, (2008), pp. 25-29.

[7] Z. C. Sha, H. B. Wu and X. T. Ren, "Second-order moment of autocorrelaton for signal detection in non-cooperative DS/SS communication”, Systems Engineering and Electronics, vol. 35, no. 8, (2013), pp. 1602-1606.

[8] Y. X. Feng, F. Liu and G. F. Pan, Editor, "New synchronization mechanism of direct sequence spread spectrum signals", National Defense Industry Press, Beijing, (2011).

[9] M. X. Guan and L. Wang, "A Novel Recognition Method for Low SNR DSSS Signals Based on FourOrder Cumulant and Eigenvalue Analysis", China Journal of Electronics, vol. 24, no. 3, (2015), pp. 2015121(1-8) 
[10] T. Q. Zhang, D. N. He and S. Chen, "Spectral correlation-based parameter estimation of BOC modulation signal”, J. Huazhong Univ. of Sci \&Tech. (Natural Science Edition), vol. 41, no. 9, (2013), pp. $11-16$.

[11] Y. Zhang, H. Wu and Y. Chen, "Period estimation of PN sequence for weak DSSS signals based on improved power spectrum reprocessing in non-cooperative communication systems", 2012 International Conference on Control Engineering and Communication Technology, (2012), pp. 924-927.

[12] J. Jäntti, S. Chaudhari and V. Koivunen, "Detection and Classification of OFDM Waveforms Using Cepstral Analysis", IEEE Transactions on Signal Processing, vol. 63, no. 16, (2015), pp. 4284-4299.

[13] P. Velavan and M. Santhi, "Design and FPGA realization of MC-CDMA system using pseudo chaotic sequence generator", International Conference on Communication and Signal Processing, (2014) November 7, pp. 498-502

[14] B. Qian, Y. X. Feng and C. S. Pan, "The Method of Parameter Estimation for TDDM-BOC Modulation Signal", Information and control, vol. 40, no. 4, (2011), pp. 459-466.

[15] Y. Zhou and T. Q. Zhang, "Period Estimation of PN Sequence for TDDM-BOC Signal in Multipath Environment", Telecommunication Engineering, vol. 55, no. 6, (2015), pp. 651- 657.

[16] V. Nir and B. Scheer, "Robust blind carrier frequency synchronisation for direct sequence spread spectrum systems", Electronic Letters, vol. 51, no. 5, (2015), pp. 427-432.

[17] S. Wu, J. Tian and W. Cui, "A Novel Parameter Estimation Algorithm for DSSS Signals Based on Compressed Sensing", China Journal of Electronics, vol. 24, no. 2, (2015), pp. 434-438.

[18] R. Yang, T. Q. Zhang and S. Shi, "Blind Estimation of Pseudo Code Period and Combination Code for BOC Signals", Telecommunication Engineering, vol. 54, no. 6, (2014), pp. 759-764.

[19] T. Q. Zhang, Y. Zhou and F. Ye, "Subcarrier type identification and parameters blind estimation of TDDM-BOC modulation signal", Xi Tong Gong Cheng Yu Dian Zi Ji Shu/Systems Engineering and Electronics, vol. 38, no. 4, (2016), pp. 922-928.

[20] Z. Y. Ma, G. X. Zhang and D. M. Bian, "A new parameter estimation method based on spectrum character in satellite communication", Applied Mechanics and Materials, Mechanics, Mechatronics, Intelligent System and Information Technology, vol. 610, (2014), pp. 921-926.

[21] J. C. Ding, L. Zhao, C. Jia And Z. B. Luo, "BDS multipath parameter estimation in the presence of impulsive noise", International Journal of Antennas and Propagation, vol. 2015, (2015).

[22] G. Wilfried and B. Michael, "Estimation of carrier and channel parameters in time-selective fading channels", IET Communications, vol. 9, no. 12, (2015), pp. 1474-1478.

[23] X. Huang, Q. Zhao and W. M. Jiang, "Frequency estimation of cyclic spectrum carrier based on compressive sampling of BPSK signal", IET Conference Publications, vol. 2015, no. CP677, (2015).
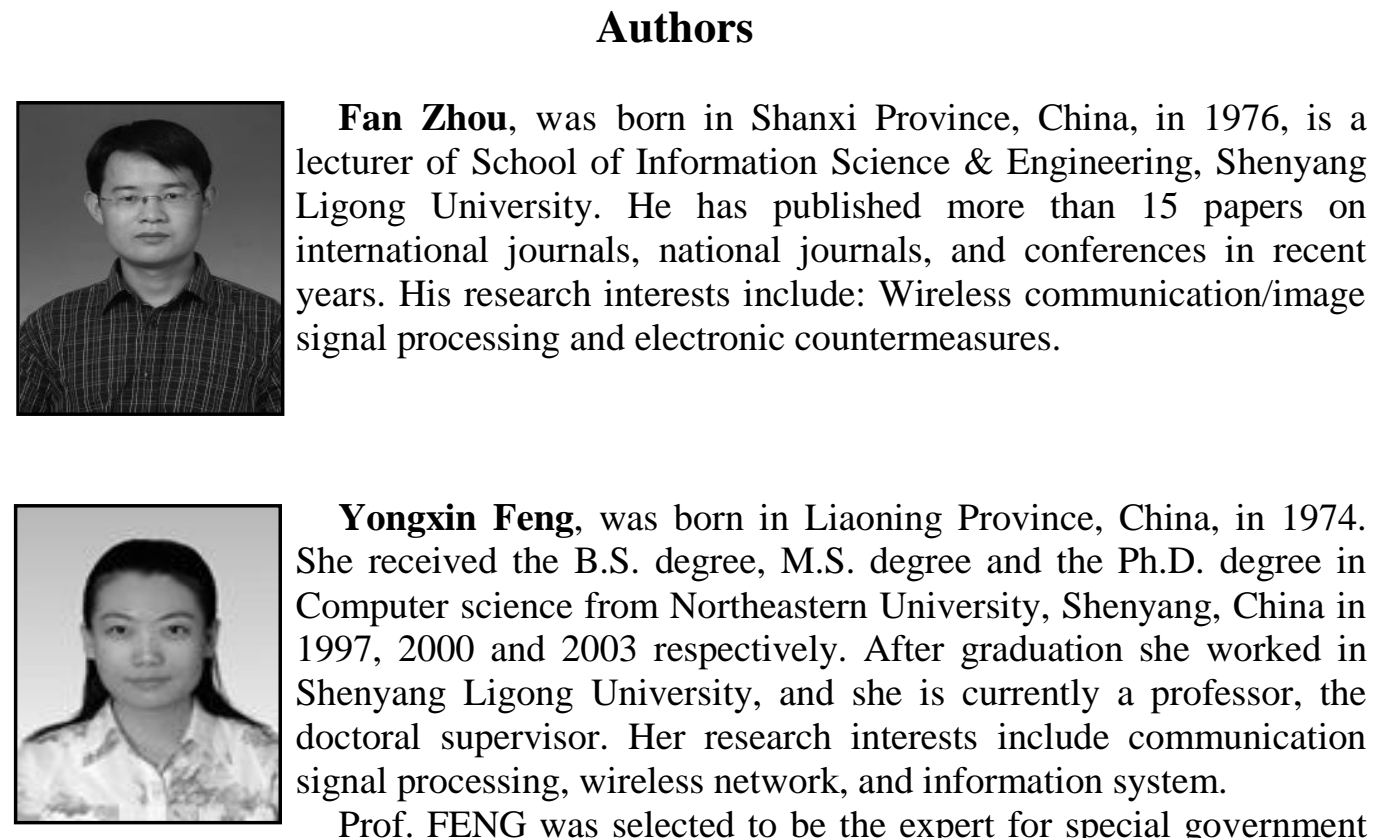

Yongxin Feng, was born in Liaoning Province, China, in 1974. She received the B.S. degree, M.S. degree and the Ph.D. degree in Computer science from Northeastern University, Shenyang, China in 1997, 2000 and 2003 respectively. After graduation she worked in Shenyang Ligong University, and she is currently a professor, the doctoral supervisor. Her research interests include communication signal processing, wireless network, and information system.

Prof. FENG was selected to be the expert for special government allowance from the State Department, Liaoning first distinguished professor, outstanding experts, winner of Education Ministry's new century excellent talents supporting plan, the number of Liaoning province new century national hundred talent project, etc.,. 


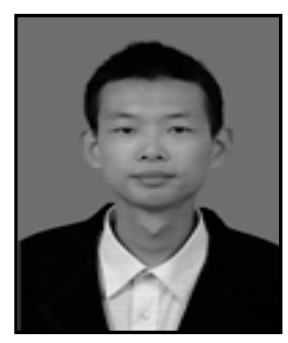

Xiaoyu Zhang, was born in Shandong Province, China, in 1988. He received the B.S. degree in Information engineering from Ludong University, Yantai, China, in 2011 and the M.S. degree in Communication and Information system from Shenyang Ligong University, Shenyang, China, in 2015. He is currently pursuing the Ph.D. degree in Armament Science and technology from Shenyang Ligong University. His research interests include spread spectrum signal processing, cognitive wireless network, and information systems. 
International Journal of Signal Processing, Image Processing and Pattern Recognition Vol. 9, No. 10, (2016) 